\title{
Rapid communication: insights into the role of extracellular vesicles during Auger radioimmunotherapy
}

Jihad Karam, Julie Constanzo, Alexandre Pichard, Laurent Gros, Joël Chopineau, Marie Morille, and JeanPierre Pouget

QUERY SHEET

This page lists questions we have about your paper. The numbers displayed at left are hyperlinked to the location of the query in your paper.

The title and author names are listed on this sheet as they will be published, both on your paper and on the Table of Contents. Please review and ensure the information is correct and advise us if any changes need to be made. In addition, please review your paper as a whole for typographical and essential corrections.

Your PDF proof has been enabled so that you can comment on the proof directly using Adobe Acrobat. For further information on marking corrections using Acrobat, please visit http://journalauthors.tandf.co.uk/production/acrobat.asp; https://authorservices.taylorandfrancis.com/how-to-correct-proofs-with-adobe/

The CrossRef database (www.crossref.org/) has been used to validate the references.

\section{AUTHOR QUERIES}

Q1 Please check the authors' names, affiliations, and corresponding details have been typeset correctly and correct if this is inaccurate.

Q2 There is no mention of (Humm et al. 1994 ) in the text. Please insert a citation in the text or delete the reference as appropriate.

Q3 Please provide the volume number for Rosenkranz et al. 2020.

Q4 Please note that the ORCID section has been created from information supplied with your manuscript submission/CATS. Please correct if this is inaccurate.

Q5 Please provide author's short biography (for each author) in not more than 50 words each to be added in the "Notes on contributors" section.

Q6 The year for "Pomplun et al. 1994" has been changed to 1987 to match the entry in the references list. Please provide revisions if this is incorrect. 


\section{Rapid communication: insights into the role of extracellular vesicles during Auger radioimmunotherapy}

Q4 Jihad Karam ${ }^{\mathrm{a}}$, Julie Constanzo ${ }^{\mathrm{a}}$, Alexandre Pichard ${ }^{\mathrm{a}}$, Laurent Gros ${ }^{\mathrm{a}}$, Joël Chopineau ${ }^{\mathrm{b}}$, Marie Morille ${ }^{\mathrm{b}}$, and

Q1 Jean-Pierre Pouget ${ }^{a}$

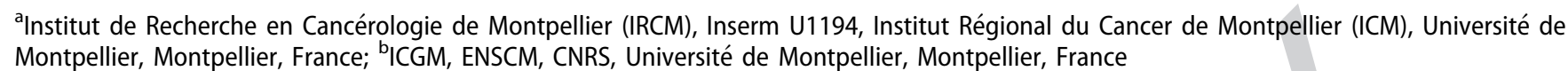

\begin{abstract}
Purpose: Non-targeted effects, including bystander and systemic effects, play a crucial role during Auger targeted radionuclide therapy. Here, we investigated whether small extracellular vesicles ( $\mathrm{sEV}$ s) produced by irradiated cells could contribute to the bystander cytotoxic effects in vitro and also to therapeutic efficacy in vivo, after their injection in tumor xenografts.

Materials and methods: B16F10 melanoma donor cells were exposed to radiolabeled antibodies (Auger radioimmunotherapy, RIT) for $48 \mathrm{~h}$ or to X-rays (donor cells). Then, donor cells were incubated with fresh medium for $2 \mathrm{~h}$ to prepare conditioned medium (CM) that was transferred onto recipient cells for bystander effect assessment, or used for sEVs enrichment. Resulting sEVs were incubated in vitro with recipient cells for determining bystander cytotoxicity, or injected in B16F10 melanoma tumors harbored by athymic and C57BL/6 mice.

Results: In vitro analysis of bystander cytotoxic effects showed that CM killed about $30-40 \%$ of melanoma cells. SEVs isolated from CM contributed to this effect. Moreover, the double-stranded DNA (dsDNA) content was increased in SEVs isolated from CM of exposed cells compared to control (not exposed), but the difference was significant only for the X-ray condition. These results were supported by immunodetection of cytosolic dsDNA in donor cells, a phenomenon that should precede dsDNA enrichment in sEVs. However, sEVs cytotoxicity could not be detected in vivo. Indeed, in athymic and in immunocompetent mice that received four intratumoral injections of sEVs (1/day), tumor growth was not delayed compared with untreated controls. Tumor growth was slightly (not significantly) delayed in immunocompetent mice treated with sEVs from X-ray-exposed cells, and significantly with SEVs purified from CM collected after $48 \mathrm{~h}$ of incubation. These results highlight the need to determine the optimal conditions, including radiation absorbed dose and sEVs collection time, to obtain the strongest cytotoxic effects.

Conclusions: This study demonstrates that $\mathrm{SEV}$ s could play a role during Auger RIT through bystander effects in vitro. No systemic effects were observed in vivo, under our experimental conditions. However, X-rays experiments showed that SEVs collection time might be influencing the nature of sEVs, a parameter that should also be investigated during Auger RIT.
\end{abstract}

\section{ARTICLE HISTORY}

Received 25 October 2020

Revised 23 June 2021

Accepted 28 June 2021

\section{KEYWORDS}

Targeted radionuclide therapy; radiotherapy; Auger electrons; exosomes; extracellular vesicles

\section{Introduction}

Atom decay via internal conversion or electronic capture creates vacancies in the orbital inner shells that can be filled by transitions of electrons from the outer shells. These transitions are accompanied by emission of X-photons or lowenergy Auger electrons, Coster-Kronig and super Coster-Kronig electrons, depending on the involved electron transitions; however, all the ejected electrons are usually called Auger electrons (AEs). Most AEs have energy below $1 \mathrm{keV}$ with very short range in tissue $(<1 \mu \mathrm{m})$ (Pomplun Q6 et al. 1987; Kassis 2004; Howell 2008) and high linear energy transfer $(4-24 \mathrm{keV} / \mu \mathrm{m})$, compared with gamma rays and beta particles $(0.2 \mathrm{keV} / \mu \mathrm{m})$. As nuclear DNA is the most sensitive target in cells, many studies investigated the best way to drive AEs into cell DNA (Rosenkranz et al. 2020).
This was satisfyingly obtained with drugs that behave like 95 chemotherapy, for example radioactive DNA base analogs 97 (e.g. 5-[(125)I]iodo-2'-deoxyuridine; [ $\left.\left.{ }^{125} \mathrm{I}\right] \mathrm{I}-\mathrm{UdR}\right) \quad$ (Kassis 98 et al. 1987). Yet, the development of more targeted 99 approaches in cancer therapy requires to target specifically 100 cancer cells. This was obtained using peptides and monoclo- 101 nal antibodies (mAbs) (Costantini et al. 2007; Pouget et al. 102 2008; Santoro et al. 2009; Aghevlian et al. 2017; Ku et al. 103 2019). However, these molecules generally recognize cancer 104 cell surface receptors and are internalized in endosomes 105 where they are degraded or recycled before they can reach 106 the nucleus (Rosenkranz et al. 2020). About 10 years ago, we 107 demonstrated in vitro and in mice that AEs display some 108 antitumor efficacy when targeted to the membrane of tumor 109 cells using mAbs. We showed that targeting iodine 125110

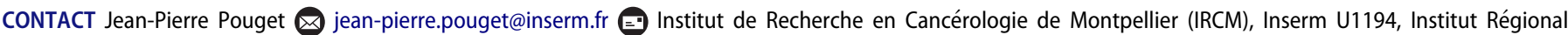
du Cancer de Montpellier (ICM), Université de Montpellier, 208 rue des apothicaires, 34298 Montpellier, France 
$\left({ }^{125} \mathrm{I}\right)$ to nuclear DNA using $\left[{ }^{125} \mathrm{I}\right] \mathrm{I}-\mathrm{UdR}$ was a more efficient approach and killed about $50 \%$ of cells compared with $15 \%$ when ${ }^{125} \mathrm{I}$-mAbs were targeted to the cell membrane. However, we also found that about $30 \%$ of cells were killed by bystander effects (Paillas et al. 2013; Paillas et al. 2016). Therefore, we concluded that bystander effects could compensate for the anticipated inferior efficacy of the absence of nuclear targeting. Moreover, AEs-labeled mAbs bypass the disadvantages of radioactive chemotherapy. We demonstrated that cell membrane-mediated targeted and non-targeted (bystander) effects were associated with lipid raft formation and subsequent activation of signaling pathways involved in cell death (Ladjohounlou et al. 2019). However, we did not investigate the nature of the factors involved in ${ }^{125} \mathrm{I}$-mAb-induced bystander effects.

Extracellular vesicles (EVs) are one of the most attractive and promising candidates identified in the last decade for participating to intercellular communications. EVs are commonly classified in three subcategories: (i) apoptotic bodies (diameter range $1-5 \mu \mathrm{m}$ ) produced during apoptosis; (ii) microvesicles $(100 \mathrm{~nm}-1 \mu \mathrm{m})$ (MVs), which bud directly from the plasma membrane; (iii) exosomes, the smallest EVs (50-150 nm), which are released by the fusion of multivesicular endosomal bodies with the plasma membrane (van Niel et al. 2018; Malloci et al. 2019). Many studies using powerful analytical tools have recently highlighted the complexity and heterogeneity of these vesicles, and revealed the existence of subtypes, depending on several parameters (cell source, state, isolation protocols, etc.) (Colombo et al. 2014; Kowal et al. 2016; Théry et al. 2018; Malloci et al. 2019). In this context, we adopted international EVs community guidelines and use the term small EVs (sEVs), rather than exosomes, as the overlapping in size, similar morphology and variable composition make the distinction of EVs type by current purification methods difficult (Lötvall et al. 2014; Théry et al. 2018).

Interestingly, EVs are produced during external exposure to X-rays as part of many cellular processes, including activation of the immune system (Al-Mayah et al. 2015; Jelonek et al. 2016; Diamond et al. 2018). However, to our knowledge, no study has assessed their role during Auger radioimmunotherapy (RIT). Here, we report preliminary results on the role of sEVs as mediators of by stander and systemic effects in melanoma cells exposed to Auger RIT or X-rays.

\section{Materials and methods}

\section{Cell lines, antibody and radiolabeling, $X$-rays}

B16F10 mouse melanoma cells (from ATCC) were grown in RPMI1640 medium supplemented with $10 \%$ fetal bovine serum (FBS) and $1 \%$ penicillin. For exosome collection, cells were incubated in Opti-MEM medium (31985070, Thermo Fisher Scientific), to avoid contamination by fetal bovine serum EVs. B16F10 cells were cultured at $37^{\circ} \mathrm{C}$ and $5 \%$ $\mathrm{CO}_{2}$, and tested regularly for mycoplasma contamination and for rodent pathogens. B16F10 cells were chosen because they are a well-established and widely used model of melanoma that allows investigating the immune system involvement in vivo (Overwijk and Restifo 2000).

The anti-mouse tyrosinase-related protein 1 (TYRP-1, TRP-1) TA99 mAb (IgG2a format) was purchased from BioXcell. TYRP-1 is the most abundant glycoprotein synthesized by melanocytes and pigmented melanoma cells, and is specific to melanocytes, and primary and metastatic melanomas (They et al. 2017). For Auger RIT, TA99 radiolabeling was performed according to the IODO-GEN (1,3,4,6-tetrachloro-3a, 6a-diphenylglycoluryl) (Sigma-Aldrich, St. Louis, MO) method ( $\left.{ }^{125} \mathrm{I}-\mathrm{TA} 99\right)$ at the specific activity of 370 $\mathrm{MBq} / \mathrm{mg}$ as previously described (Paillas et al. 2016). TA99 antibody radiolabeling was performed according to the IODO-GEN (1,3,4,6-tetrachloro-3a, 6a-diphenylglycoluryl) (Sigma-Aldrich, St. Louis, MO) method (Pouget et al. 2008). ${ }^{125}$ I-TA99 specific activity was $370 \mathrm{MBq} / \mathrm{mg}$ of protein, and immunoreactivity was $60-95 \%$. All experiments used ${ }^{125}$ I-TA99 activities $\leq 4 \mathrm{MBq} / \mathrm{mL}$. Cells were also exposed to $\mathrm{X}$-rays (XenX $\mathrm{X}^{\circledR}$, Xstrahl, $\left.225 \mathrm{kV} ; 2 \mathrm{~Gy} / \mathrm{min}\right)$.

\section{Preparation of conditioned medium from X-ray- and RIT-treated cells}

The protocol shown in Figure 1(A) was followed. Typically, 50-300 B16F10 donor cells/well were seeded in 6-well plates containing $2 \mathrm{~mL}$ of medium. The following day, cells were exposed to ${ }^{125}$ I-TA99 $(0-4 \mathrm{MBq} / \mathrm{mL})$ (RIT) for $48 \mathrm{~h}$ or to $0.5 \mathrm{~Gy} \mathrm{X}$-rays. The dose of $0.5 \mathrm{~Gy} \mathrm{X}$-rays was chosen as bystander effects have been shown to occur at dose below 1 Gy (Nagasawa and Little 1992; Belyakov et al. 2001; Prise and O'Sullivan 2009). Then, cells were washed with Dulbecco's phosphate-buffered saline (PBS) twice before addition of fresh Opti-MEM ${ }^{\circledR}$ medium (for sEVs collection) or RMPI 1640 medium with 10\% FBS (for bystander experiments) for $2 \mathrm{~h}$. The choice of $2 \mathrm{~h}$ incubation was based on previous experiments using other cell lines showing that conditioned medium (CM) from Auger RIT-treated cells was cytotoxic and genotoxic (Paillas et al. 2013, 2016). Then, CM was transferred to recipient cells for bystander effect measurement or used for sEVs isolation.

\section{Determination of direct and bystander cytotoxicity induced by X-rays and Auger RIT}

$\mathrm{CM}$ was added to untreated recipient B16F10 cells to determine the bystander effects, while fresh non-radioactive medium was added to donor cells after RIT/X-ray exposure to determine the direct effects. Colonies were counted after 10 days of culture as described in (Paillas et al. 2013) to determine clonogenic survival.

\section{In vitro generation of small extracellular vesicles (sEVs)}

sEVs were purified from $\mathrm{CM}$ samples at $4{ }^{\circ} \mathrm{C}$ with the protocol described by (Théry et al. 2006) (Figure 1(A)). First, CM samples were centrifuged at $300 \times g$ for $5 \mathrm{~min}$ and then at $2000 \times \mathrm{g}$, for $20 \mathrm{~min}$ to discard living and dead cells, 
(A)

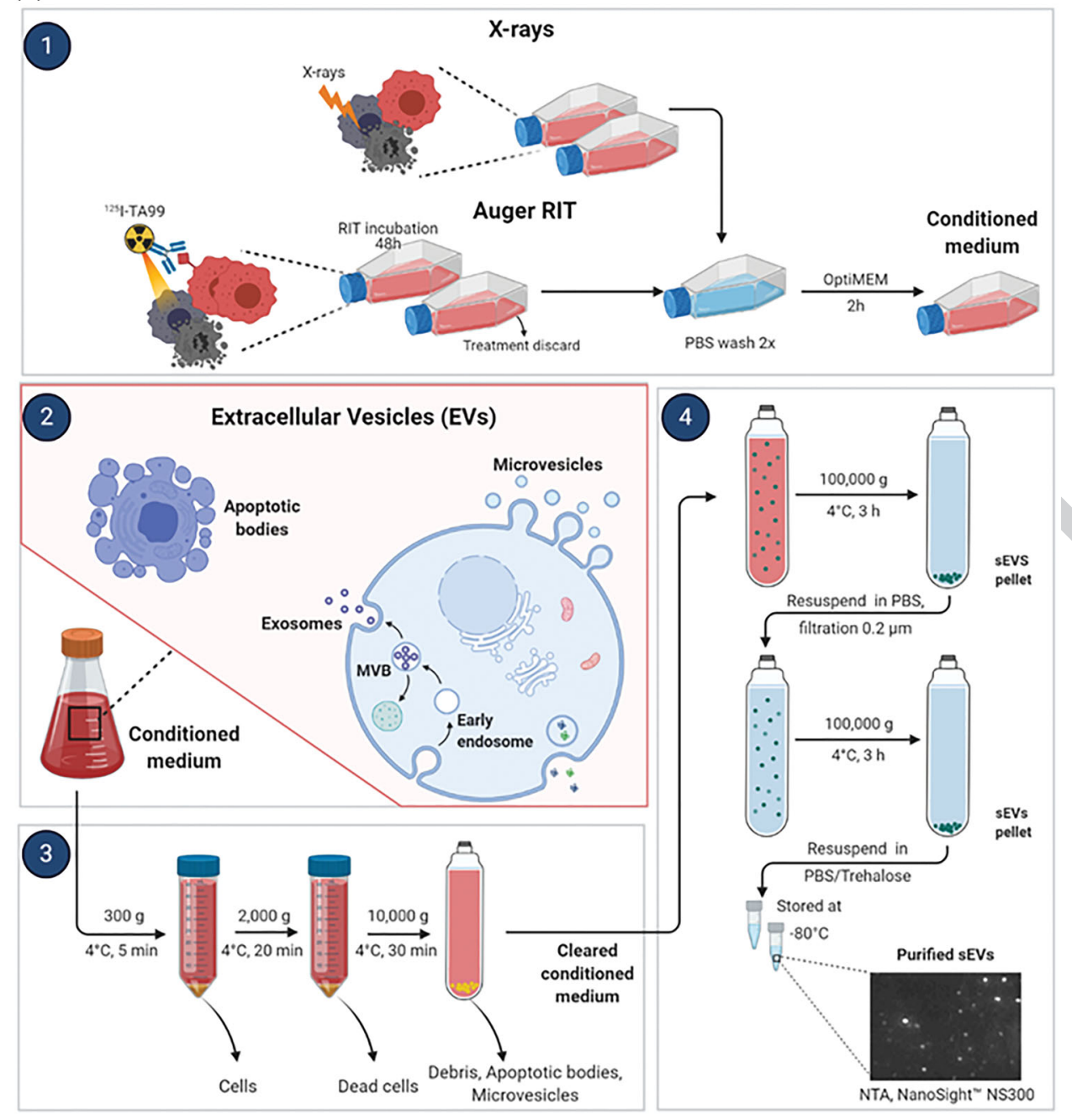

(B) B16F10 - Auger RIT (125I-TA99)

(C) B16F10-X-rays

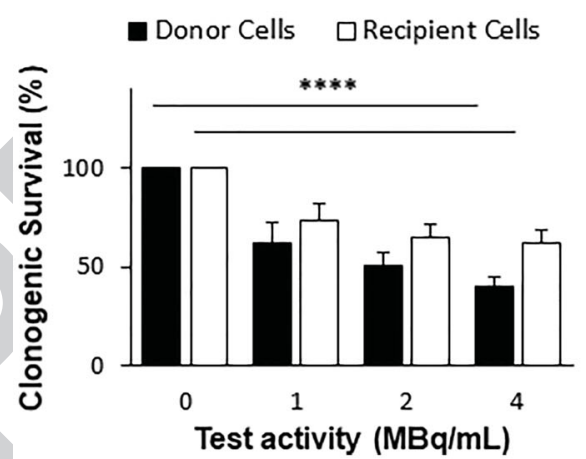

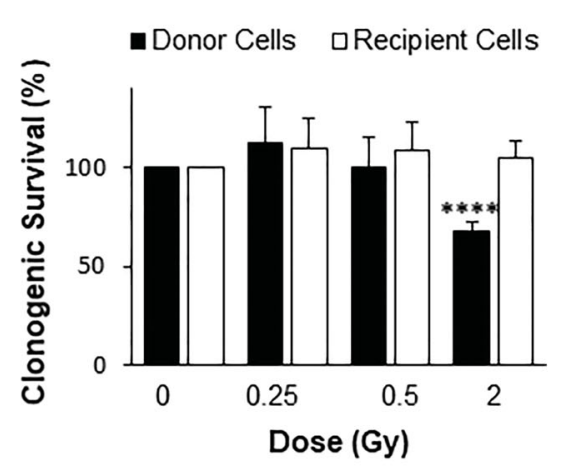

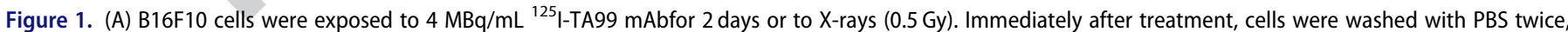

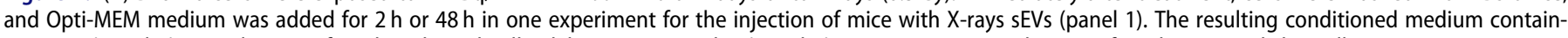

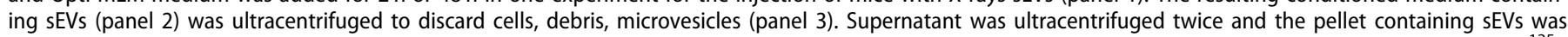

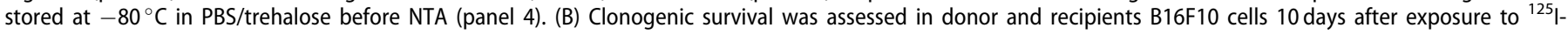

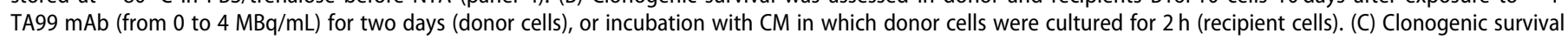

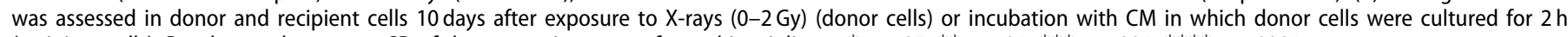
(recipient cells). Results are the mean \pm SD of three experiments performed in triplicate. ${ }^{*} p<.05,{ }^{*} p<.01, * * * p<.001,{ }^{*} * * * p<.0001$.

respectively, contained in the pellet. Supernatants were then ultracentrifuged at $10,000 \times \mathrm{g}$ for $30 \mathrm{~min}$ to discard largest vesicles, and the supernatant was then ultracentrifugated at $100,000 \times \mathrm{g}$ for $3 \mathrm{~h}$ to pellet $\mathrm{sEV}$ s fraction. The supernatant was discarded and sEVs were resuspended with PBS, filtered through $0.22 \mu \mathrm{m}$ filters before a second ultracentrifugation time under the same conditions. Finally, sEVs-containing pellets were resuspended in $200-600 \mu \mathrm{L}$ of $\mathrm{PBS} / 25 \mathrm{mM}$ trehalose and stored at $-80^{\circ} \mathrm{C}$ (following Le Saux et al. 2020). Cells were counted following the CM collection. 


\section{Extracellular vesicles characterization}

sEVs concentration (number of particles per $\mathrm{mL}$ ) and size were determined by nanoparticle tracking analysis (NTA) using a NanoSight ${ }^{\mathrm{TM}}$ NS300instrument (MalvernPanalytical, Malvern, UK). Suspensions were diluted with particle-free PBS to obtain a particle concentration in the $1 \times 10^{7}-1 \times 10^{9}$ particles $/ \mathrm{mL}$ range, as recommended by manufacturer. Measurements were performed with a $405 \mathrm{~nm}$ laser. sEVs suspensions were analyzed using the NanoSight NTA 3.2 software following a tailored script: temperature was set at $25^{\circ} \mathrm{C}$, syringe pump at $40 \mathrm{AU}$ (arbitrary unit), three videos of $60 \mathrm{~s}$ were recorded. Videos were recorded with a camera level set to 16 and analyzed with a detection threshold set to 6 . The number of sEVs released by one cell can be estimated by dividing the total number of particles measured by NTA by the total number of cells.

The expression of some proteins generally associated with sEVs expressed by endosomal sourced vesicles (exosomes) was determined by western blotting using antibodies against ALIX (E6P9B; rabbit mAb, 92880S, Cell Signaling Tec), ADAM10 (ab1997, Abcam, Paris, France), TSG101(14497-1AP, Proteintech), and CD81 (D5O2Q;rabbit mAb, 10037, Cell Signaling Technology Inc, Berverly, MA USA). Briefly, $1 \times 10^{8}$ EV particles were lysed in RIPA buffer supplemented with $1 \% \mathrm{NaVO}_{4}$, phenylmethylsulfonyl fluoride (PMSF), and Protease Inhibitor Cocktail (sc-24948A, Santa Cruz Biotechnology, Heidelberg, Germany) on ice for $20 \mathrm{~min}$. Then, samples were heated at $95^{\circ} \mathrm{C}$ for $5 \mathrm{~min}$ after addition of 4X Laemmli Sample Buffer (1610747, Bio-Rad, Marnes-la-Coquette, France) with $\beta$-mercaptoethanol (M3148, Sigma-Aldrich, St. Quentin Fallavier, France). Proteins were separated by SDS-PAGE and transferred to nitrocellulose membranes (170-4159, Bio-Rad) that were incubated with primary antibodies (1:1000) overnight. As there are not housekeeping proteins clearly identified so far for sEVs, Western blot analysis aimed at providing qualitative information only. Immune reactions were detected with horseradish peroxidase-conjugated goat anti-rabbit secondary antibodies $(1: 1000,1 \mathrm{~h}) \quad(7074 \mathrm{~S}$, Cell Signaling Technology, Danvers, MA, USA) and the enhanced chemiluminescence (ECL) detection system (1705060, Bio-Rad).

\section{Immunodetection of cytosolic double-stranded DNA (dsDNA) in B16F10 cells}

Based on the protocol by (Spada et al. 2019), B16F10 cells grown in $12 \mathrm{~mm}$ diameter coverslips were fixed with $4 \%$ PFA and permeabilized for $7 \mathrm{~min}$. After blocking nonspecific binding sites using a $1 \%$ bovine serum albumin (BSA)/PBS buffer, cells were incubated with an anti-dsDNA antibody (ab27156, Abcam), diluted to $1: 1000$ in $1 \%$ BSA/PBST, at $4{ }^{\circ} \mathrm{C}$ overnight. After three washes in $1 \mathrm{X}$ PBS, cells were incubated with a goat anti-mouse IgG Alexa Fluor ${ }^{\circledR} 488$ at room temperature for $1 \mathrm{~h}$. After three washes in $1 \mathrm{X}$ PBS, coverslips were mounted on a slide using one drop of Vectashield ${ }^{\circledR}$ antifade mounting medium with DAPI (H1200 , Vector Laboratories). Images were acquired at $40 \times$ magnification using a Zeiss ${ }^{\circledR}$ Apotome.2 microscope.

\section{Quantification of sEVs dsDNA content}

Based on the method by (Spada et al. 2020), sEVs dsDNA was extracted using the QIAamp DNA Mini Kit (51304, Qiagen, Courtaboeuf, France), and its concentration was measured using the fluorescent dsDNA-binding dye QuantiFluor $^{\circledR}$ ONE dsDNA System (Promega Corporation, Madison, WI, USA). The amount of sEVs dsDNA (in $\mathrm{fg}$ ) was estimated relative to the dsDNA concentration $(\mathrm{fg} / \mu \mathrm{L})$ measured with the standard lambda dsDNA curve and corrected by the dilution factor and the initial volume of purified sEVs per cell.

\section{Cytotoxicity of Auger RIT- and X-ray-generated SEVs}

About 50-70 B16F10 donor cells/well were seeded in 6-well plates containing $2 \mathrm{~mL}$ of medium before incubation with $0-4 \times 10^{8}$ particles $/ \mathrm{mL}$ isolated from CM samples of Auger RIT- or X-ray-treated cells. Clonogenic assays were performed as before.

\section{Mice}

431

432

Female athymic nude (Crl:NU(NCr)-Foxn1nu) and C57BL/ 6J mice (6-8-week-old; Charles River) were acclimated for 1 week. They were housed at $22^{\circ} \mathrm{C}$ and $55 \%$ humidity with a light-dark cycle of $12 \mathrm{~h}$, and food and water ad libitum. Body weight was determined weekly, and mice were clinically examined throughout the study. Animal experiments were performed in compliance with the French government guidelines and the INSERM standards for experimental animal studies (agreement B34-172-27). They were approved by the Institut de Recherche Cancérologie de Montpellier (IRCM/INSERM) and the Languedoc Roussillon region (CEEA LR France No. 36) (reference number: 1353-9340) ethics committees for animal experimentation. $5 \times 10^{4}$ B16F10 melanoma cells in $100 \mu \mathrm{L}$ of culture medium (without FBS) were inoculated subcutaneously in mice. Amounts of sEVs intratumorally administered in mouse were based on Matsumoto et al. study (Matsumoto et al. 2017) in which $10 \mu \mathrm{g}$ exosomes were split in 3 injections. This value was drastically reduced to take into account constraints due to radioactive handling. Finally, when tumors reached $20 \mathrm{~mm}^{3}$, mice ( $n=8$ mice/group) received four consecutive daily intratumoral injections of $4 \times 10^{8} \mathrm{sEVs}(0.01 \mu \mathrm{g})$ in $50 \mu \mathrm{L}$ $\mathrm{PBS} /$ trehalose each injection, purified from cells exposed to $0.5 \mathrm{~Gy} \mathrm{X}$-rays or to $4 \mathrm{MBq} / \mathrm{mL}{ }^{125} \mathrm{I}$-anti-TA99, or vehicle alone (PBS/trehalose). Tumor growth was monitored every two days by caliper measurement, and mice were euthanized when the tumor reached a volume of $2000 \mathrm{~mm}^{3}$. Tumor volume was calculated using the following formula: length$\times$ width $\times$ width $/ 2$.

\section{Statistics}

Data were expressed as mean \pm standard deviation (SD) for in vitro experiments, and as mean \pm standard error of mean (SEM) for in vivo experiments. For clonogenic survival,
433

434

435

436

437

438

439

440

441

442

443

444

445

446

447

448

449

450

451

452

453

454

455

456

457

458

459

460

461

462

463

464

465

466

467

468 
comparison between groups were performed using nonparametric Mann-Whitney $t$-tests. A $p$-value $\leq .05$ was considered significant. Statistical analyses were done with GraphPad Prism ${ }^{\circledR}$ 8.4.3.

\section{Results}

\section{Bystander effects and EVs production are induced during Auger RIT}

Exposure to ${ }^{125} \mathrm{I}$-TA99 mAb strongly decreased clonogenic survival of B16F10 melanoma cells in an activity-dependent manner $(40 \pm 7 \%$ at $4 \mathrm{MBq} / \mathrm{mL} ; p<.0001$ compared with untreated cells) (Figure 1(B)). Similarly, clonogenic survival of recipient $\mathrm{B} 16 \mathrm{~F} 10$ cells incubated in $\mathrm{CM}$ decreased to $62 \pm 9 \% \quad(p<.0001)$. Clonogenic survival of donor cells exposed to $2 \mathrm{~Gy} \mathrm{X}$-rays decreased to $68 \pm 6 \%(p<.0001)$.
Conversely, no bystander cytotoxicity was observed in 528 recipient cells incubated with CM from cells exposed to $2 \mathrm{G} 529$ $\mathrm{X}$-rays $(105 \pm 9 \%$, Figure $1(\mathrm{C}))$. We determined in a previous 530 study using a nonspecific ${ }^{125} \mathrm{I}$ - $\mathrm{PX} \mathrm{mAb}$ that soft $\mathrm{X}$-rays pro- 531 duced by ${ }^{125} \mathrm{I}$ do not contribute to cell killing and that 532 resulting mean nucleus absorbed dose is about $0.2 \mathrm{~Gy} 533$ (Pouget et al. 2008).

To test the role of sEVs in the observed bystander 535 effects, exosomes were purified from CM samples from 536 cells exposed to Auger RIT or X-rays (Figure 2(A)). sEVs 537 number per cell varied between 2 and 56 and between 1538 and 91 for Auger RIT- and X-ray-treated cultures. Size dis- 539 tribution (NTA) was similar for both conditions, with a 540 mean diameter of $128 \pm 12 \mathrm{~nm}$ and $122 \pm 8 \mathrm{~nm}$, respectively 541 (Figure 2(A)). Western blotting confirmed the presence of 542 the sEVs markers ALIX, ADAM10, TSG101 and CD81 543 (Figure 2(B)).
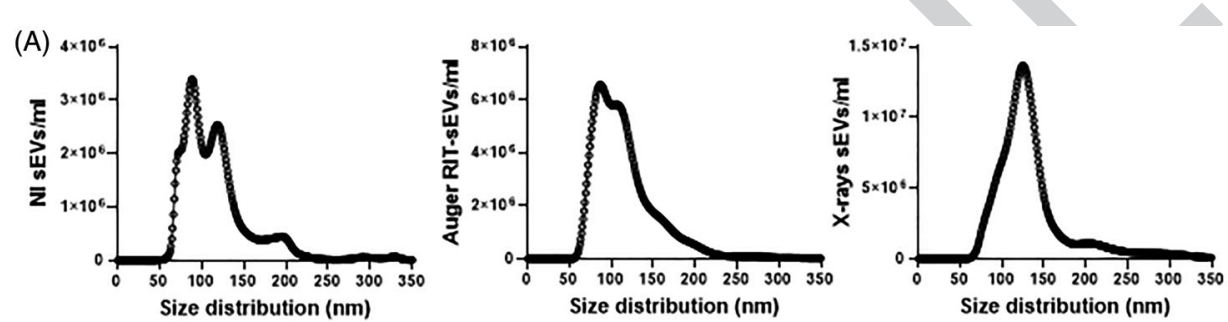

\begin{tabular}{|c|c|c|c|}
\cline { 2 - 4 } \multicolumn{1}{c|}{} & NI sEVs & Auger RI sEVs & X-rays sEVs \\
\hline Average size $(\mathrm{nm})$ & $121 \pm 3$ & $128 \pm 12$ & $122 \pm 8$ \\
\hline Mode $(\mathrm{nm})$ & $102 \pm 21$ & $97 \pm 10$ & $90 \pm 25$ \\
\hline Nb of exosomes released/cell & $6-28$ & $2-56$ & $1-91$ \\
\hline
\end{tabular}

(B)
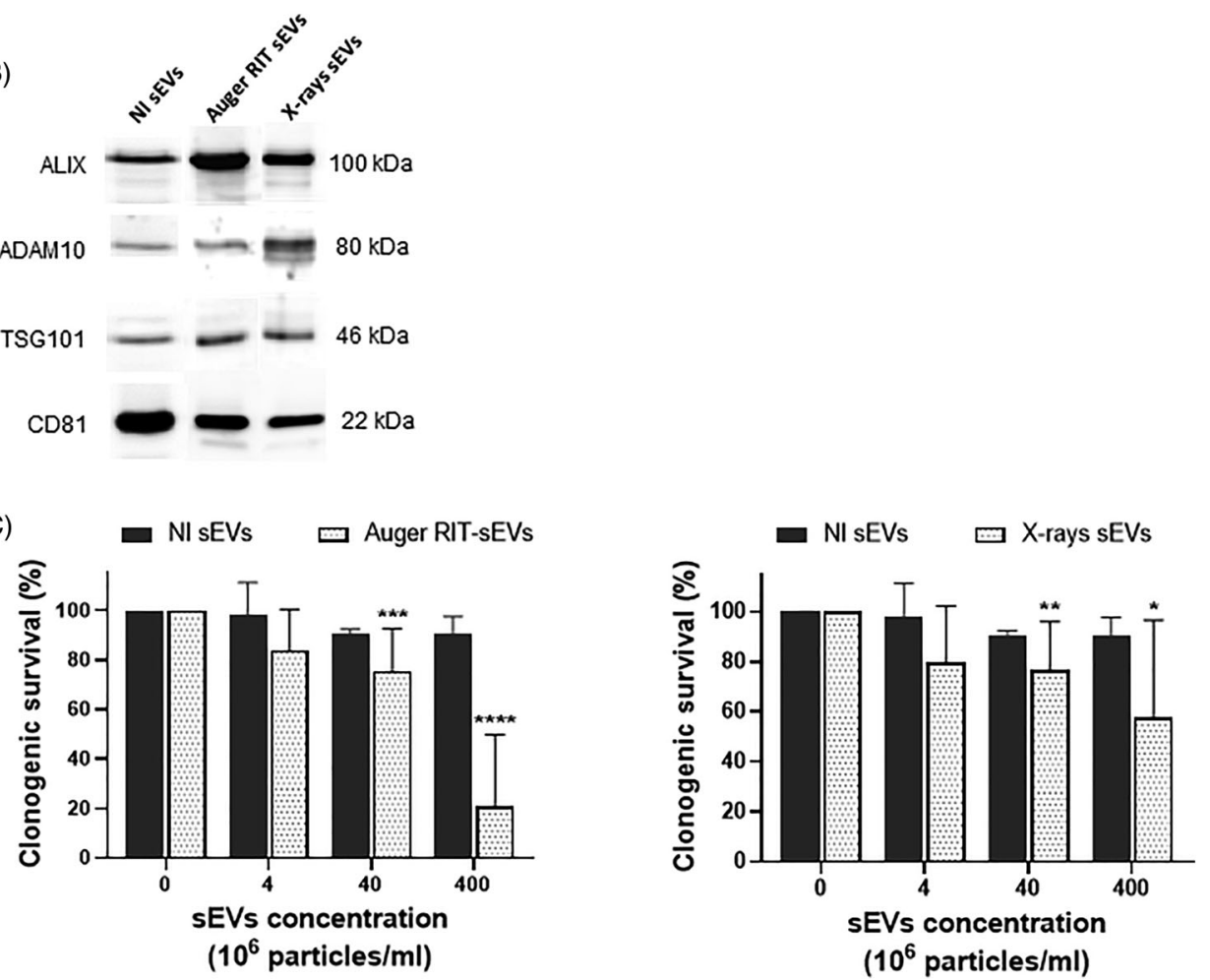

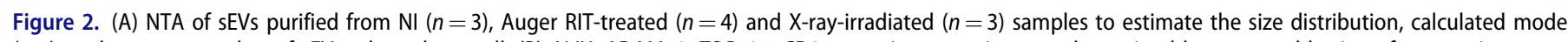

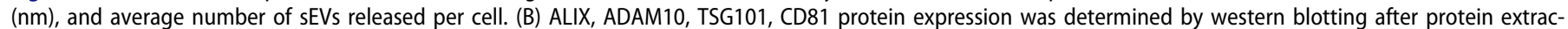

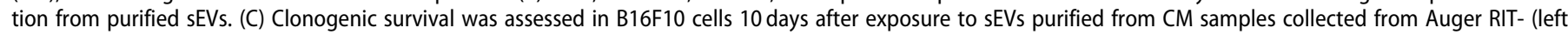




\section{X-ray- and Auger RIT-generated sEVs are cytotoxic in vitro}

Then, B16F10 cells were incubated with various concentrations of purified sEVs $\left(0-400 \times 10^{6}\right.$ particles $\left./ \mathrm{mL}\right)$. Clonogenic survival was significantly decreased in a dosedependent manner, and dropped to $67 \pm 32 \%(p=0.047)$ and $21 \pm 29 \%(p<.0001)$ with $400 \times 10^{6} \mathrm{sEVs} / \mathrm{mL}$ from CM of Xray- and Auger RIT-treated cells, respectively (Figure 2(C)).

\section{Enrichment of sEVs in dsDNA}

Accumulation of cytosolic dsDNA was shown to be progressive during the first $48 \mathrm{~h}$ following cell exposure to Auger
RIT (Figure 3(A)). Next, quantification of dsDNA in purified sEVs (Figure 3(B)) indicated that its concentration was slightly increased in sEVs isolated from Auger RIT-treated cells compared with sEVs from non-irradiated cells (NI) $(0.55 \pm 0.18$ versus $0.33 \pm 0.16$ fg per cell; $p=.06)$ Conversely, it was strongly increased in sEVs from X-rayirradiated cells $(1.23 \pm 0.44$ fg per cell; $p=.0007)$.

\section{Systemic effects of X-ray- and Auger RIT-generated sEVs}

Finally, immunocompetent and immunosuppressed (lacking T-cells) mice harboring B16F10 cell xenografts were treated with sEVs isolated from CM of Auger RIT- and
646

647

648

649

650

651

652

653

654

655

656

657

658

659

660

661

662

663

664

665

B16F10, CTRL

MERGE

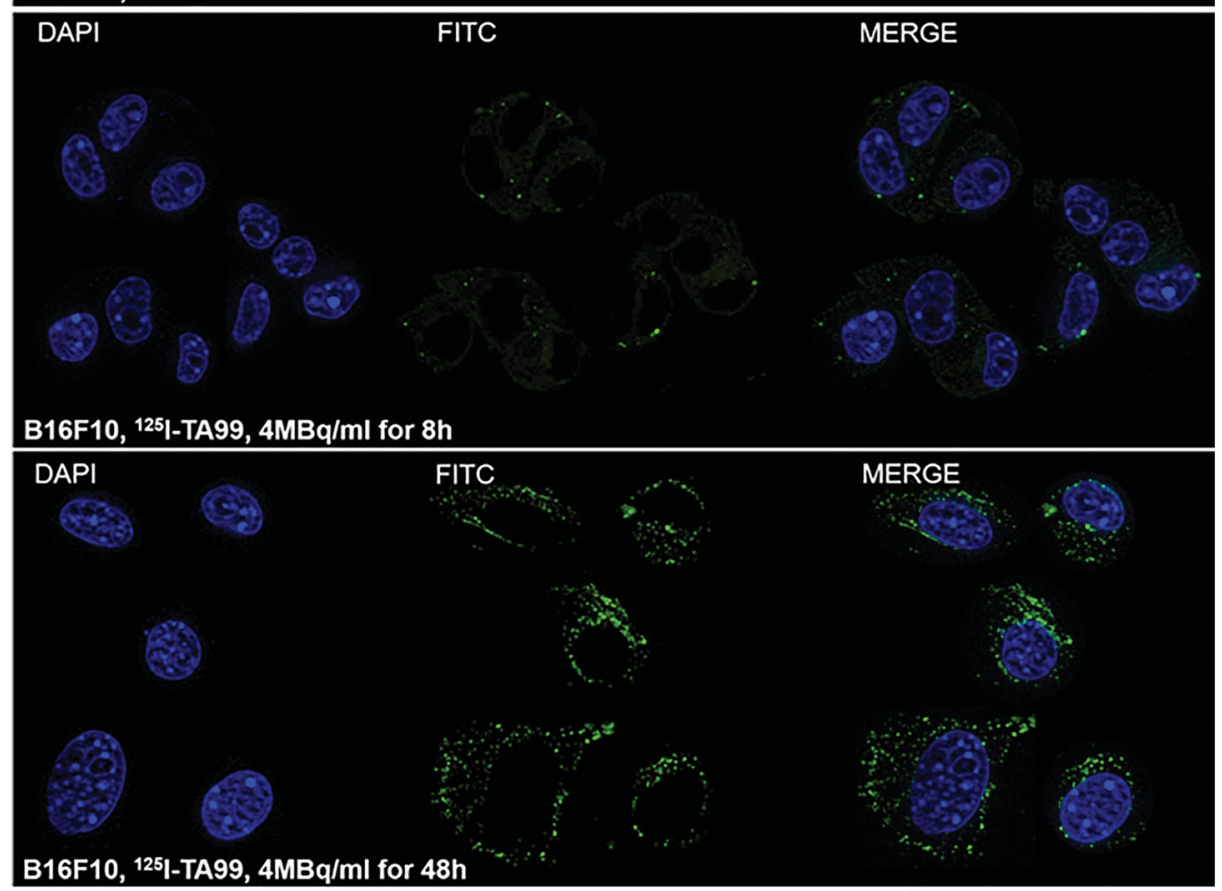

$\star * *$

(B)

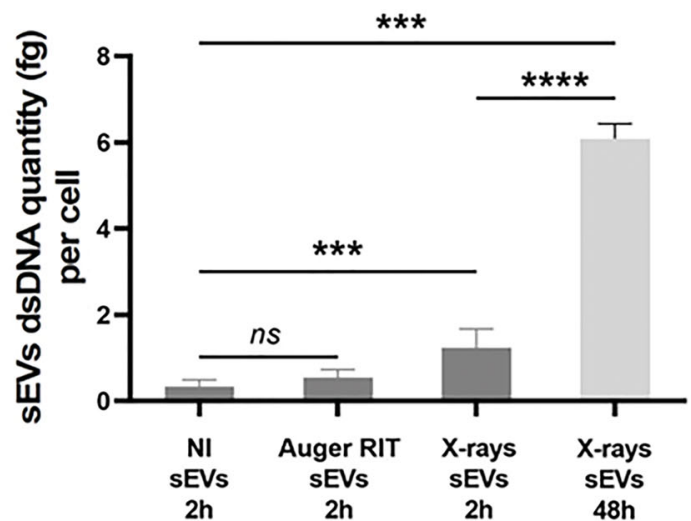

Figure 3. (A) Cytosolic dsDNA was detected in cells at $8 \mathrm{~h}$ (middle panels) and $48 \mathrm{~h}$ (lower panels) after Auger-RIT initiation. (B) dsDNA was quantified in sEVs isolated from CM samples collected from Auger RIT- or X-ray-exposed cells. Results are the mean \pm SD of four experiments performed in triplicate. CTRL, non-exposed cells (upper panels). ${ }^{*} p<.05,{ }^{* *} p<.01,{ }^{* * *} p<.001,{ }^{* * * *} p<.0001$. Nl: not irradiated. Experiments were performed two times in quadruplicates.
666

667

668

669

670

671

672

673

674

675

676

677

678

679

680

681

682

683

684

685

686

687

688

689

690

691

692

693

694

695 
705

706

707

708 B\&W in

709

710

711

712

713

714

715

716

717

718

719

720

721

722

723

724

725

726

727

728

729

730

731

732

733

734

735

736

737

738

739

740

741

742

743

744

745

746

747

748

749

750

751

752

753

754

755

756

757

758

759

760

761

762

763
(A)

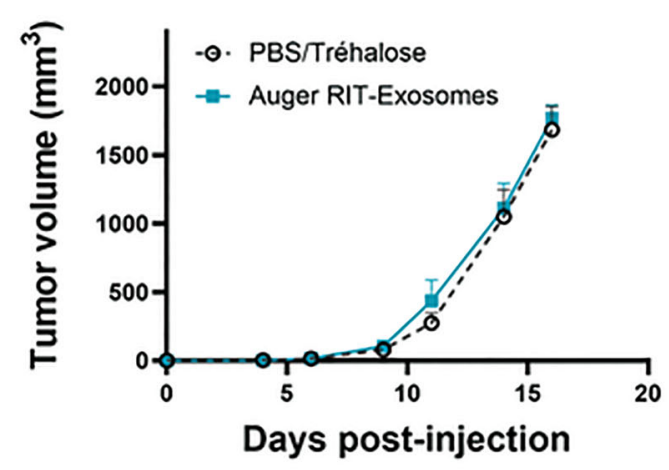

Nude mice

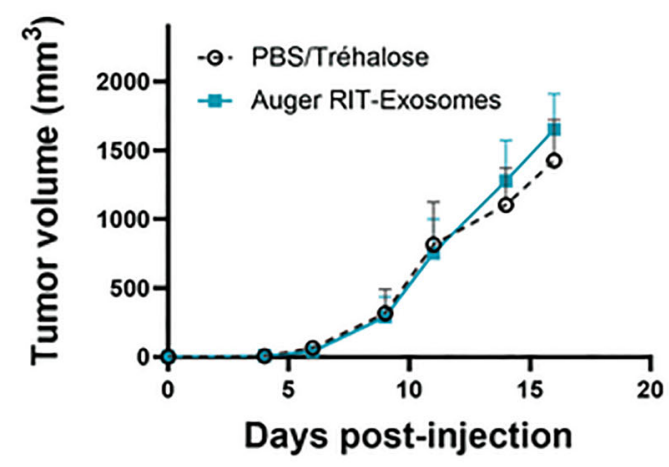

(C)
(B)

WT mice

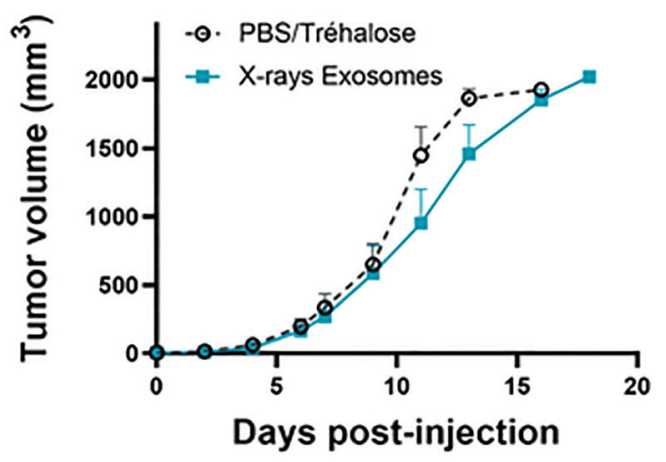

Nude mice

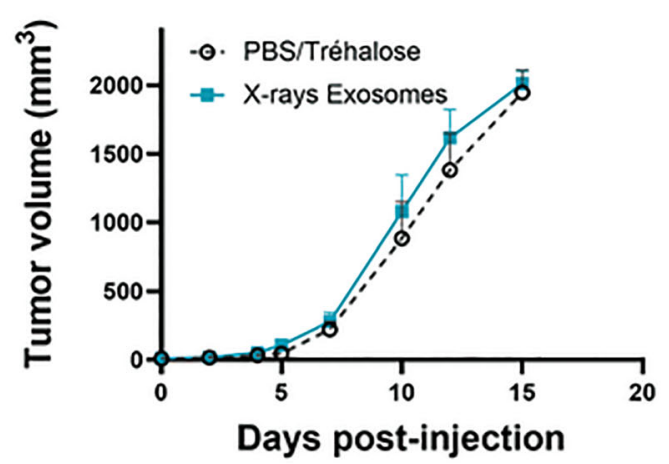

764

765

766

767

768

769

770

771

772

773

774

775

776

777

778

779

780

781

782

783

784

785

786

787

788

789

790

791

792

793

794

795

796

797

798

799

800

801

802

803

Figure 4. In vivo therapeutic efficacy of $s E V s$ in immunocompetent (C57BL/6) and immunosuppressed (athymic nude) mice. (A) sEvs isolated from CM samples of 804 Auger RIT-treated $(4 \mathrm{MBq} / \mathrm{mL}$ ) cells. (B) $\mathrm{sEVs}$ isolated from CM samples of X-ray-irradiated cells ( $0.5 \mathrm{~Gy})$. (C) sEVs isolated from CM samples collected from X-ray-irra- 805 diated cells $(0.5 \mathrm{~Gy})$ after $48 \mathrm{~h}$ incubation. Tumor growth was monitored as a function of the time post-tumor injection.

delay tumor growth compared with control (vehicle) (Figure $4(\mathrm{~A}, \mathrm{~B})$ ), although, sEVs from $\mathrm{CM}$ of $\mathrm{X}$-ray-exposed cells slightly reduced tumor growth in immunocompetent mice between 9 and 15 days post-treatment (Figure 4(B)). This effect was enhanced by injection of sEVs isolated from CM collected after $48 \mathrm{~h}$ incubation with X-ray-irradiated cells (Figure $4(\mathrm{C})$ ) (preliminary data, $n=3$ mice per group). Corresponding enrichment of sEVs in dsDNA was then about $6.1 \pm 0.3 \mathrm{fg} / \mathrm{cell}$. This strong efficacy of intratumoral injection of sEVs collected $48 \mathrm{~h}$ after onset of irradiation has also been observed in another RIT model using a different type of radiation (unpublished results).

\section{Discussion}

811

SEVs secreted from all cell types that participate in local and distal intercellular communication, and thus are attractive candidates for explaining bystander and systemic cytotoxic effects (Al-Mayah et al. 2015; Jelonek et al. 2016; de Araujo Farias et al. 2018). Here, we investigated whether small sEVs released in the supernatant of murine melanoma B16F10 cells exposed to Auger RIT contributed to the in vitro bystander cytotoxic effects. We also tested their in vivo cytotoxic effects after injection in tumor xenografts in immunosuppressed and immunocompetent mice. To compare our 821 results with previous data from our group, we used the 822 
same in vitro protocol ((Paillas et al. 2013; Piron et al. 2014; Paillas et al. 2016; Ladjohounlou et al. 2019). Donor cells were exposed to ${ }^{125} \mathrm{I}$-TA99 mAb for $48 \mathrm{~h}$, and CM was prepared by incubating donor cells with exosome-free fresh medium for $2 \mathrm{~h}$.

SEVs obtained from CM samples from Auger RIT- and $\mathrm{X}$-ray-exposed cells, were shown to express ALIX, ADAM10, TSG101 and CD81, proteins generally associated with sEVs expressed by endosomal sourced vesicles (exosomes). These sEVs caused cytotoxic effects in vitro at a concentration of $4 \times 10^{8}$ particles $/ \mathrm{mL}$. The extrapolation between the concentration of purified sEVs and their concentration in CM samples used for bystander experiments is not straightforward. Indeed, sEVs were purified from large CM volumes through successive ultracentrifugation steps (Figure 1(A)). Therefore, it is likely that their concentration was much higher than in $\mathrm{CM}$ samples. This could explain why bystander effects were not observed with $\mathrm{CM}$ from cells exposed to $0.5 \mathrm{~Gy} \mathrm{X}$-rays (Figure $1(\mathrm{C})$ ), whereas clonogenic survival was significantly reduced by sEVs purified from such CM (Figure 2(C)).

However, sEVs cytotoxicity was not confirmed in mice harboring B16F10 cell xenografts which may suggest that they do not induce bystander or systemic effects in vivo. This could be due to the strong aggressiveness of melanoma tumors, which finally overwhelms the cytotoxicity of 4 injections of sEVs. The aggressiveness of the latter tumors was also observed during Auger and alpha RIT as high activities, as compared with other carcinoma models, were required to delay tumor growth (data not shown). Moreover, we did not check the fate of intratumorally injected sEVs. Matsumoto et al. demonstrated that sEVs administered in B16BL6 tumors remained within the tumor up to $48 \mathrm{~h}$ post-injection (Matsumoto et al. 2017). In another study, Smyth et al. found that intravenously-injected vesicles were cleared rapidly while those administered in tumors were still detected at $24 \mathrm{~h}$ (Smyth et al. 2015). Conversely, we know that during Auger RIT, radiolabeled antibodies remain bound to tumors for several days and this stimulation could therefore be able to generate continuous induction of $\mathrm{sEVs}$ secretion by tumor cells. We assumed that the dsDNA contained in sEVs (Figure 3(B)), known to act as danger damage-associated molecular pattern (DAMP) signals, would be determinant in triggering the bystander and systemic responses. However, it must be kept in mind that bystander and systemic cytotoxic effects of sEVs may involve totally different mechanisms.

The role of dsDNA and its shuttling via exosomes during external radiotherapy has been highlighted in recent studies (Vanpouille-Box et al. 2017; Diamond et al. 2018). Cytosolic dsDNA acts as an activator of the DNA sensor cGAS and of its downstream effector STING that will lead to type I-IFN release and T-cell recruitment and activation and ultimately to systemic tumor rejection.

We hypothesize that dsDNA concentration in sEVs from $\mathrm{CM}$ of Auger RIT-exposed cells was not enough to observe an anti-tumor immune response. In agreement, sEVs isolated from $\mathrm{CM}$ of X-ray-exposed cells, in which dsDNA concentration was higher, showed a slight tumor growth delay in vivo. This effect was higher with sEVs collected from CM collected after $48 \mathrm{~h}$ incubation with X-ray-irradiated cells which was accompanied by a higher dsDNA enrichment. It must be noted that the size $(122 \pm 8 \mathrm{~nm}$ vs $129 \pm 8 \mathrm{~nm}$ ) and expression of sEVs biomarkers (TSG101, CD81, ALIX, ADAM10) was not modified when CM incubation time was increased. However the number of collected sEVs was significantly increased $(\times 30 \pm 25)$ (data not shown). This suggests that dsDNA abundance in sEVs might be influenced by the incubation duration before $\mathrm{CM}$ collection. It must be noted that Auger RIT mechanisms of action strongly differ from that of X-rays. This could explain different yield and kinetic induction of dsDNA between the two forms of irradiation. Indeed, the whole cell and the nucleus are directly irradiated by X-rays in a homogeneous way. Conversely, ${ }^{125} \mathrm{I}-\mathrm{mAbs}$ targeting the cell membrane mostly deliver the dose to the cell membrane (Arnaud et al. 2016). This energy deposit leads to the formation of ceramideenriched large domain (lipid rafts) that participate in downstream signaling cascades involving $\mathrm{p} 38, \mathrm{JNK}, \mathrm{NF}-\mathrm{kB}$ and reactive oxygen species production. Therefore, the formation of DNA double-strand breaks (DSBs) and micronuclei (Piron et al. 2014; Paillas et al. 2016), which possibly will contribute to dsDNA presence in sEVs, is delayed compared with X-rays. Another factor modulating DNA DSBs is that during RIT, lesions are repaired at the same time as they are generated (Piron et al. 2014) and it is necessary to wait for the radiation absorbed dose to accumulate to overcome DNA repair. Piron found that DNA DSBs were increased only after $24 \mathrm{~h}$ post-Auger RIT initiation in a colorectal cancer model (Piron et al. 2014). Then, unrepaired or misrepaired DNA would lead to the formation of micronuclei, that in turn would release their DNA into cytosol before being incorporated into sEVs (Figure 3(C)) (Hintzsche et al. 2017; Takahashi et al. 2017). Then, if the yield of micronuclei depends on the cell repair capacity, it depends also on the radiation absorbed dose, a parameter that was not determined here and should be further investigated. The absorbed dose can also play a role by modulating the activation of the DNA exonuclease TREX1, leading to the digestion of cytoplasmic dsDNA and subsequent tempering of the immune response. Indeed, Vanpouille-Box et al. (2017) found in TSA cells that after a single dose of X-rays higher than $12 \mathrm{~Gy}$, cytosolic dsDNA was cleared by TREX1, precluding activation of the cGAS pathway to induce IFN-1, and thus abolishing the anti-tumor immune response. Therefore, targeted radionuclide dosimetry is a crucial parameter that needs to be considered.

\section{Conclusion}

We showed that sEVs isolated from CM of Auger-RITexposed cells are associated with about $30-40 \%$ bystander cytotoxic effects in vitro. Cytoplasmic dsDNA progressively increased in B16F10 cells during Auger-RIT, and was enriched in sEVs purified from CM samples of exposed cells. However, in vivo use of sEVs for cancer treatment showed no therapeutic effects under our conditions. Based on our X-ray results, it is not possible to exclude that other 
conditions (absorbed radiation dose and sEVs collection time) would not modulate differently the production of sEVs differently during RIT Auger together with their cytotoxic effects.

\section{Disclosure statement}

The authors report no conflicts of interest. The authors alone are responsible for the content and writing of the paper.

\section{Funding}

Q5 This work was supported by, SIRIC Montpellier Cancer Grant INCa_Inserm_DGOS_12553, French National Research Agency as part of the Investissements d'Avenir program (ANR-10-LABX-53-01) and Cancéropôle Grand Sud Ouest, fondation ARC pour la recherche sur le cancer.

\section{ORCID}

Jihad Karam (D) http://orcid.org/0000-0002-8651-8681

\section{References}

Aghevlian S, Boyle AJ, Reilly RM. 2017. Radioimmunotherapy of cancer with high linear energy transfer (LET) radiation delivered by radionuclides emitting $\alpha$-particles or Auger electrons. Adv Drug Deliv Rev. 109:102-118.

Al-Mayah A, Bright S, Chapman K, Irons S, Luo P, Carter D, Goodwin E, Kadhim M. 2015. The non-targeted effects of radiation are perpetuated by exosomes. Mutat Res. 772:38-45.

Arnaud FX, Paillas S, Pouget JP, Incerti S, Bardiès M, Bordage MC. 2016. Complex cell geometry and sources distribution model for Monte Carlo single cell dosimetry with iodine 125 radioimmunotherapy. Nucl Instrum Methods Phys Res, Sect B. 366:227-233.

Belyakov OV, Malcolmson AM, Folkard M, Prise KM, Michael BD. 2001. Direct evidence for a bystander effect of ionizing radiation in primary human fibroblasts. Br J Cancer. 84(5):674-679.

Colombo M, Raposo G, Théry C. 2014. Biogenesis, secretion, and intercellular interactions of exosomes and other extracellular vesicles. Annu Rev Cell Dev Biol. 30(1):255-289.

Costantini DL, Chan C, Cai Z, Vallis KA, Reilly RM. 2007. In-labeled trastuzumab (Herceptin) modified with nuclear localization sequences (NLS): an Auger electron-emitting radiotherapeutic agent for HER2/neu-amplified breast cancer. J Nucl Med. 48(8):1357-1368.

de Araujo Farias V, O'Valle F, Serrano-Saenz S, Anderson P, Andrés E, López-Peñalver J, Tovar I, Nieto A, Santos A, Martín F, et al. 2018. Exosomes derived from mesenchymal stem cells enhance radiotherapy-induced cell death in tumor and metastatic tumor foci. Mol Cancer. 17(1):122.

Diamond JM, Vanpouille-Box C, Spada S, Rudqvist N-P, Chapman JR, Ueberheide BM, Pilones KA, Sarfraz Y, Formenti SC, Demaria S. 2018. Exosomes shuttle TREX1-sensitive IFN-stimulatory dsDNA from irradiated cancer cells to DCs. Cancer Immunol Res. 6(8): 910-920.

Hintzsche H, Hemmann U, Poth A, Utesch D, Lott J, Stopper H. 2017. Fate of micronuclei and micronucleated cells. Mutat Res. 771:85-98.

Howell RW. 2008. Auger processes in the 21st century. Int J Radiat Biol. 84(12):959-975.

Humm JL, Howell RW, Rao DV. 1994. Dosimetry of Auger-electronemitting radionuclides: report no. 3 of AAPM Nuclear Medicine
Jelonek K, Widlak P, Pietrowska M. 2016. The influence of ionizing 1000 radiation on exosome composition, secretion and intercellular com- 1001 munication. Protein Pept Lett. 23(7):656-663.

Kassis AI, Sastry KS, Adelstein SJ. 1987. Kinetics of uptake, retention, and radiotoxicity of $125 \mathrm{IUdR}$ in mammalian cells: implications of localized energy deposition by Auger processes. Radiat Res. 109(1): 78-89.

2004. The amazing world of auger electrons. Int T Radiat 1006 Biol. 80(11-12):789-803.

Kowal J, Arras G, Colombo M, Jouve M, Morath JP, Primdal-Bengtson B, Dingli F, Loew D, Tkach M, Théry C. 2016. Proteomic comparison defines novel markers to characterize heterogeneous populations of extracellular vesicle subtypes. Proc Natl Acad Sci U S A. 113(8): E968-E977. USA.

Ku A, Facca VJ, Cai Z, Reilly RM. 2019. Auger electrons for cancer 1012 therapy - a review. EJNMMI Radiopharm Chem. 4(1):27.

Ladjohounlou R, Lozza C, Pichard A, Constanzo J, Karam J, Le Fur P, Deshayes E, Boudousq V, Paillas S, Busson M, et al. 2019. Drugs that modify cholesterol metabolism alter the p38/JNK-mediated targeted and nontargeted response to alpha and auger radioimmunotherapy. Clin Cancer Res. 25(15):4775-4790.

1013

1014

1015

1016 G, Bertrand-Michel J, Dubois E, George S, Faklaris O, et al. 2020 1018 Post-production modifications of murine mesenchymal stem cell 1019 (mMSC) derived extracellular vesicles (EVs) and impact on their 1020 cellular interaction. Biomaterials. 231:119675.

1021

Lötvall J, Hill AF, Hochberg F, Buzás EI, Di Vizio D, Gardiner C, Gho 1022 YS, Kurochkin IV, Mathivanan S, Quesenberry P, et al. 2014. 1023 Minimal experimental requirements for definition of extracellular 1024 vesicles and their functions: a position statement from the International Society for Extracellular Vesicles. J Extracell Vesicles. 3(1):26913.

Malloci M, Perdomo L, Veerasamy M, Andriantsitohaina R, Simard G, 1027 Martínez MC. 2019. Extracellular vesicles: mechanisms in human 1028 health and disease. Antioxid Redox Signal. 30(6):813-856.

Matsumoto A, Takahashi Y, Nishikawa M, Sano K, Morishita M, 1030 Charoenviriyakul C, Saji H, Takakura Y. 2017. Accelerated growth 1030
of B16BL6 tumor in mice through efficient uptake of their own exo- 1031 somes by B16BL6 cells. Cancer Sci. 108(9):1803-1810.

Nagasawa H, Little JB. 1992. Induction of sister chromatid exchanges 1033 by extremely low doses of alpha-particles. Cancer Res. 52(22): 1034 6394-6396.

Overwijk WW, Restifo NP. 2000. B16 as a mouse model for human melanoma. Curr Protoc Immunol [Internet]. 39(1). [accessed 2021 Apr 19] https://onlinelibrary.wiley.com/doi/10.1002/0471142735. im2001s39.

Paillas S, Boudousq V, Piron B, Kersual N, Bardiès M, Chouin N, 1039 Bascoul-Mollevi C, Arnaud F-X, Pèlegrin A, Navarro-Teulon I, et al. 1040 2013. Apoptosis and p53 are not involved in the anti-tumor efficacy 1041 $\begin{array}{ll}\text { of 125I-labeled monoclonal antibodies targeting the cell membrane. } & 1042 \\ \text { Nucl Med Biol. 40(4):471-480. } & \end{array}$

Paillas S, Ladjohounlou R, Lozza C, Pichard A, Boudousq V, Jarlier M, 1043 Sevestre S, Le Blay M, Deshayes E, Sosabowski J, et al. 2016. 1044 Localized irradiation of cell membrane by Auger electrons is cyto- 1045 toxic through oxidative stress-mediated nontargeted effects. Antioxid 1046 Redox Signal. 25(8):467-484.

Piron B, Paillas S, Boudousq V, Pèlegrin A, Bascoul-Mollevi C, Chouin N, Navarro-Teulon I, Pouget J-P. 2014. DNA damage-centered signaling pathways are effectively activated during low dose-rate Auger radioimmunotherapy. Nucl Med Biol. 41 Suppl:e75-e83.

Pomplun E, Booz J, Charlton DE 1987. A Monte Carlo simulation of Auger cascades. Radiat Res. 111(3):533-552.

Pouget J-P, Santoro L, Raymond L, Chouin N, Bardiès M, BascoulMollevi C, Huguet H, Azria D, Kotzki P-O, Pèlegrin M, et al. 2008. Cell membrane is a more sensitive target than cytoplasm to dense 1054 ionization produced by Auger electrons. Radiat Res. 170(2):192-200. 1055 Prise KM, O’Sullivan JM. 2009. Radiation-induced bystander signalling 1056 in cancer therapy. Nat Rev Cancer. 9(5):351-360. 


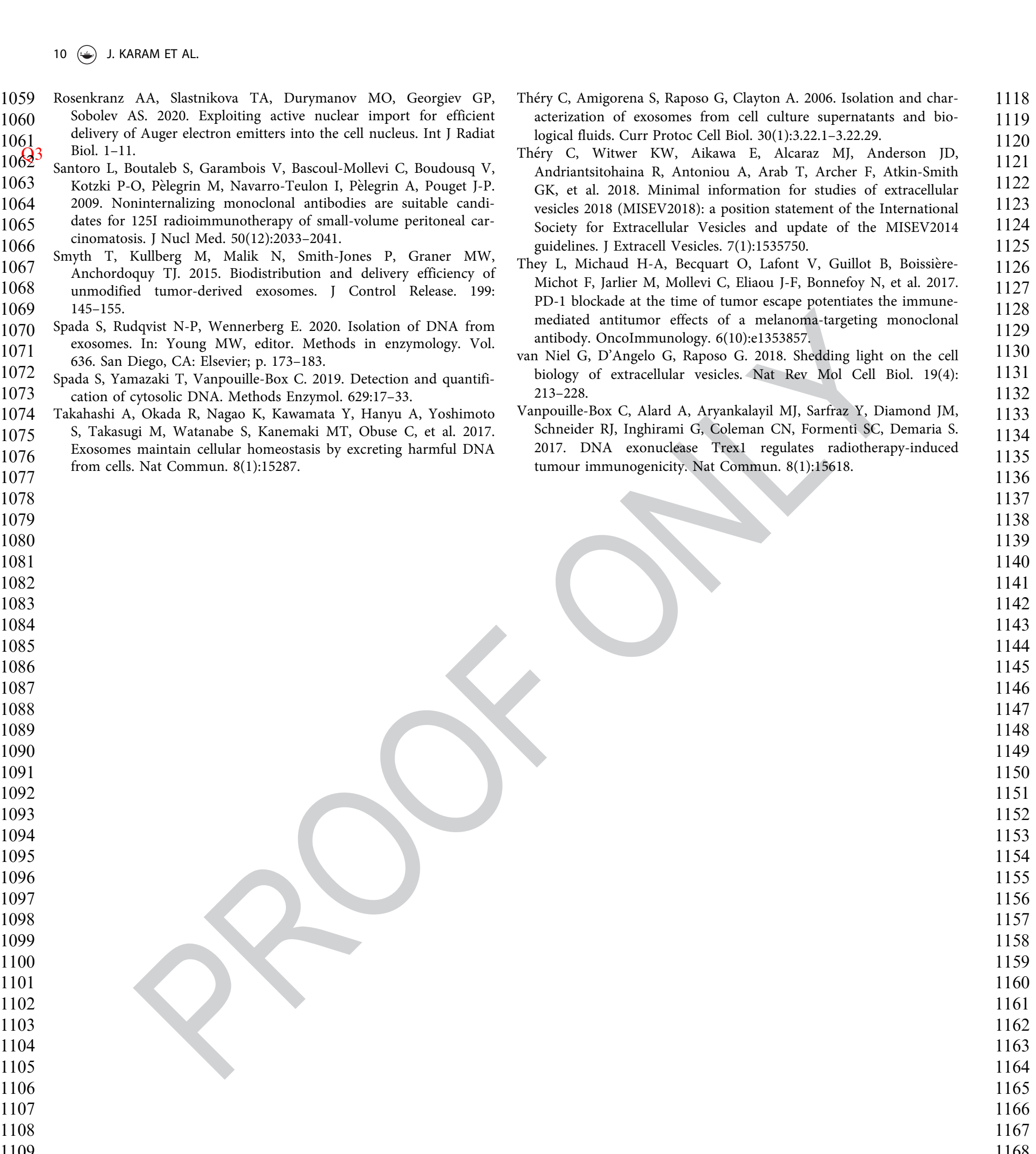

